\section{Response: Renal dosing of allopurinol results in suboptimal gout care by T Neogi et al}

We sincerely appreciate the interest shown by Dr T Neogi and colleagues $^{1}$ concerning our 2016 revised European League Against Rheumatism (EULAR) recommendations for the management of gout. ${ }^{2}$

Dr T Neogi and colleagues raised concern with the ninth item, in which we recommend to adjust the maximum dosage of allopurinol according to the creatinine clearance $(\mathrm{CrCl})$ in order to decrease the risk of severe cutaneous allergic reactions (SCARs).

First, we would like to emphasise that these recommendations were written from a European perspective. In many countries in Europe, regulatory agencies require this adjustment, an important point we took into account for the elaboration of this recommendation. Furthermore, in addition to rheumatologists, the task force included general practitioners, who manage the vast majority of people with gout, patients with gout who are on urate-lowering treatment, and evidence-based medicine (EBM) experts. Therefore, the recommendations reflect perspectives from multiple stakeholders and follow the principles of EBM by examining all types of evidence (patient views, expert opinion and experience, research evidence) in the realisation that each has its strengths and weaknesses, and it is only when all align that we get close to 'accepted best practice'.

Second, assessing the benefits and the risks of administering allopurinol to patients with chronic kidney disease (CKD) is difficult. The literature that addresses the safety of allopurinol use in patients with CKD is predominantly retrospective. After an extensive literature review, we concluded that the level of evidence to support that a dose escalation strategy is safe in patients with CKD is low. For instance, in the quoted paper by Lisa Stamp, ${ }^{3}$ the authors examined the effects of increasing the dosage of allopurinol above the recommended dose based on the $\mathrm{CrCl}$. Safety was analysed on the basis of just 45 patients, and given that the incidence of allopurinol-induced SCARs is about $0.7 / 1000$ patient-years in allopurinol initiators, ${ }^{4}$ one can easily conclude that this study was insufficiently powered to draw any firm conclusion. Furthermore, it is important to note that patients enrolled in this study had been tolerating CrCl-based doses of allopurinol for at least a month prior to titration, because it is known that the risk of SCARs occurrence is more common in the weeks following initiation. ${ }^{5}$

Third, as mentioned by Dr T Neogi, it is true that we lack clear evidence that restriction of allopurinol maintenance dose in patients with CKD might decrease the risk of SCARs. It would be wise also to remind that we also lack evidence that a starting dose of $1.5 \mathrm{mg}$ per unit of estimated glomerular filtration rate (eGFR) $\left(\mathrm{mL} / \mathrm{min} / 1.73 \mathrm{~m}^{2}\right)$ might reduce the occurrence of SCARs. Of note, the sole prospective case-control study, ${ }^{6}$ not cited by $T$ Neogi, clearly showed that renal failure was significantly associated with the delayed clearance of plasma oxypurinol, which might have antigenic properties to stimulate cytotoxic T lymphocytes. ${ }^{6}$ Moreover, in this study, renal failure increased the risk of SCARs and was correlated with a poor prognosis, in particular mortality. In addition, the starting dose, found to be associated with SCARs in a retrospective study, ${ }^{7}$ was not associated in this prospective study with an increased risk of severe allergic reactions. Finally, in subjects with severe renal impairment (eGFR $<30 \mathrm{~mL} / \mathrm{min} /$ $1.73 \mathrm{~m}^{2}$ ), there was no significant difference in the values of initial dose/eGFR between allopurinol-SCAR and tolerant controls, contrary to what was found previously. ${ }^{7}$
Therefore, there remains uncertainty surrounding the risk/ benefit ratio of allopurinol in patients with CKD, and it is unlikely that we will have prospective longitudinal data to allow determination of the appropriate starting dose and whether we can or cannot safely increase the dose in patients with CKD. We discussed in depth this issue with patients who were part of the task force, and all of them agreed, as did the general practitioners, that we should apply a precautionary principle. Thus, we recommend to initiate allopurinol with a maximum starting dose of $100 \mathrm{mg}$ daily and to keep a conservative approach for the maintenance dose to be adapted to the $\mathrm{CrCl}$, as did the British Society of Rheumatology ${ }^{8}$ and the last EULAR recommendation. ${ }^{9}$

Fourth, we disagree that this recommendation will impair the quality of care for patients with CKD. On the contrary, we strongly believe that this will facilitate the management of hyperuricaemia in these difficult-to-treat patients. As shown in figure 2 , we recommend that if the allowed allopurinol dosage does not achieve the predefined urate target levels $(5$ or $6 \mathrm{mg} / \mathrm{dL}$ ), a switch to febuxostat or a combination therapy with an old or recent uricosuric ${ }^{10}$ should be undertaken. Recent findings indicate that febuxostat remains safe and effective in patients with eGFRs as low as $15 \mathrm{~mL} / \mathrm{min},{ }^{11}{ }^{12}$ and that there is no crossreactivity between febuxostat and allopurinol. ${ }^{13}$ Thus, advocating that limiting the allopurinol dosage in patients with CKD will result in suboptimal management is incorrect, because there are now alternatives to consider-this negative assumption is based on a study published in $2006,{ }^{14}$ well before the availability of febuxostat and the novel uricosuric lesinurad. ${ }^{10}$

To conclude, we believe that initiating patients with $\mathrm{CKD}$ with a maximum of $100 \mathrm{mg}$ allopurinol daily, adapting the maintenance dose to the $\mathrm{CrCl}$ and offering therapeutic alternatives, if necessary, such as febuxostat or a combination of allopurinol and a uricosuric, should both decrease the risk and severity of SCARs and increase the quality of care.

\section{Pascal Richette, ${ }^{1,2}$ Michael Doherty, ${ }^{3}$ Eliseo Pascual, ${ }^{4}$ Thomas Bardin ${ }^{5}$}

${ }^{1}$ INSERM U1132 and University Paris-Diderot, Paris, France

${ }^{2}$ Department of Rheumatology, Hopital Lariboisiere, Paris, 75010, île-de-France, France

${ }^{3}$ Academic Rheumatology, University of Nottingham, Nottingham, UK

${ }^{4}$ Department of Rheumatology, Hospital General Universitario de Alicante, Alicante, Spain

${ }^{5}$ Department of Rheumatology, Hôpital Lariboisière, Paris, France

Correspondence to Professor Pascal Richette, INSERM U1132 and University Paris-Diderot, Paris, France; pascal.richette@lrb.aphp.fr

Contributors PR wrote the draft, and MD, EP and TB critically revised it. Competing interests None declared.

Provenance and peer review Commissioned; internally peer reviewed.

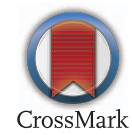

To cite Richette P, Doherty M, Pascual E, et al. Ann Rheum Dis 2017;76:e2.

Received 13 October 2016

Accepted 14 October 2016

Published Online First 3 November 2016

\section{S Linked}

http://dx.doi.org/10.1136/annrheumdis-2016-210352

Ann Rheum Dis 2017;76:e2. doi:10.1136/annrheumdis-2016-210356 


\section{REFERENCES}

1 Neogi T, Dalbeth N, Stamp L, et al. Renal dosing of allopurinol results in suboptimal gout care. Ann Rheum Dis Published Online First: 31 Aug 2016. doi:10.1136/annrheumdis-2016-210352

2 Richette P, Doherty M, Pascual E, et al. 2016 updated EULAR evidencebased recommendations for the management of gout. Ann Rheum Dis Published Online First: 25 Jul 2016. doi: 10.1136/annrheumdis-2016209707

3 Stamp LK, O'Donnell $\mathrm{J}$, Zhang $\mathrm{M}$, et al. Using allopurinol above the dose based on creatinine clearance is effective and safe in patients with chronic gout, including those with renal impairment. Arthritis Rheum 2011;63: 412-21.

4 Kim SC, Newcomb C, Margolis D, et al. Severe cutaneous reactions requiring hospitalization in allopurinol initiators: a population-based cohort study. Arthritis Care Res (Hoboken) 2012;65:578-84.

5 Stamp LK, Day RO, Yun J. Allopurinol hypersensitivity: investigating the cause and minimizing the risk. Nat Rev Rheumatol 2016;12:235-42.

6 Chung WH, Chang WC, Stocker SL, et al. Insights into the poor prognosis of allopurinol-induced severe cutaneous adverse reactions: the impact of renal insufficiency, high plasma levels of oxypurinol and granulysin. Ann Rheum Dis 2015;74:2157-64.

7 Stamp LK, Taylor WJ, Jones PB, et al. Starting dose is a risk factor for allopurinol hypersensitivity syndrome: a proposed safe starting dose of allopurinol. Arthritis Rheum 2012;64:2529-36.
8 Jordan KM, Cameron JS, Snaith M, et al. British Society for Rheumatology and British Health Professionals in Rheumatology guideline for the management of gout. Rheumatology (Oxford) 2007;46:1372-4.

9 Zhang W, Doherty M, Bardin T, et al. EULAR evidence based recommendations for gout. Part II: Management. Report of a task force of the EULAR Standing Committee for International Clinical Studies Including Therapeutics (ESCISIT). Ann Rheum Dis 2006;65:1312-24.

10 Saag KG, Fitz-Patrick D, Kopicko J, et al. Lesinurad combined with allopurinol: randomized, double-blind, placebo-controlled study in gout subjects with inadequate response to standard of care allopurinol (a US-based Study). Arthritis Rheumatol Published Online First: 26 Aug 2016. doi: 10.1002/art.39840

11 Quilis N, Andres M, Gil S, Febuxostat for patients with gout and severe chronic kidney disease: which is the appropriate dosage? comment on the article by Saag et al. Arthritis Rheumatol 2016;68:2563-4.

12 Saag $K G$, Whelton A, Becker MA, et al. Impact of febuxostat on renal function in gout patients with moderate-to-severe renal impairment. Arthritis Rheumatol 2016;68:2035-43.

13 Bardin T, Chales G, Pascart T, et al. Risk of cutaneous adverse events with febuxostat treatment in patients with skin reaction to allopurinol. A retrospective, hospital-based study of 101 patients with consecutive allopurinol and febuxostat treatment. Joint Bone Spine 2016;83:314-17.

14 Dalbeth N, Kumar S, Stamp L, et al. Dose adjustment of allopurinol according to creatinine clearance does not provide adequate control of hyperuricemia in patients with gout. J Rheumatol 2006;33:1646-50. 